\title{
Corrigendum to "Simulating Radiotherapy Effect in High-Grade Glioma by Using Diffusive Modeling and Brain Atlases”
}

\author{
Alexandros Roniotis, ${ }^{1,2}$ Kostas Marias, ${ }^{1}$ Vangelis Sakkalis $\left(\mathbb{D},{ }^{1}\right.$ Georgios C. Manikis, ${ }^{1}$ and \\ Michalis Zervakis $\mathbb{( i D}^{2}$ \\ ${ }^{1}$ Institute of Computer Science, Foundation For Research and Technology-Hellas, 70013 Heraklion, Crete, Greece \\ ${ }^{2}$ Department of Electronic \& Computer Engineering, Technical University of Crete, 73100 Chania, Greece
}

Correspondence should be addressed to Alexandros Roniotis; alexandros.roniotis@gmail.com

Received 17 November 2016; Accepted 7 December 2017; Published 28 February 2018

Copyright ( 2018 Alexandros Roniotis et al. This is an open access article distributed under the Creative Commons Attribution License, which permits unrestricted use, distribution, and reproduction in any medium, provided the original work is properly cited.

In the article titled "Simulating Radiotherapy Effect in High-Grade Glioma by Using Diffusive Modeling and Brain Atlases" [1], there was a typographic error in formula number (11). We would like to thank Borasi and Nahum [2] for identifying this error. However, the correct formula should be as follows.

$$
\begin{aligned}
& f(c) \\
& = \begin{cases}\left(\rho *\left(1-e^{-a R(t)-\beta R(t) r(t)}\right)\right) c \frac{c_{m}-c}{c_{m}}, & t \in \text { therapy, } \\
\rho c \frac{c_{m}-c}{c_{m}}, & t \notin \text { therapy. }\end{cases}
\end{aligned}
$$

In addition, we would like to differentiate our work from the strict definition of the Linear Quadratic model. Therefore, we propose changing the name of our model from "Linear Quadratic" model to "Modified Linear Quadratic" model as presented in Wahl et al. [3] cited in the article as reference [43] or we can use the term " $\alpha-\beta$ model." Therefore, the model name should be changed throughout the article.

\section{References}

[1] A. Roniotis, K. Marias, V. Sakkalis, G. C. Manikis, and M. Zervakis, "Simulating radiotherapy effect in high-grade glioma by using diffusive modeling and brain atlases," Journal of Biomedicine and Biotechnology, vol. 2012, Article ID 715812, 9 pages, 2012.

[2] G. Borasi and A. E. Nahum, "Comment on "simulating radiotherapy effect in high-grade glioma by using diffusive modeling and brain atlases"," BioMed Research International, vol. 2015, Article ID 801057, 1 page, 2015.

[3] S. Wahl, T. D. C. Foletto, and G. Feldmann, "A mathematical model for the estimation of treatment cost in cancer radiotherapy," in Proceedings of the 2009 3rd Southern Conference on Computational Modeling, MCSUL 2009, pp. 77-81, November 2009. 


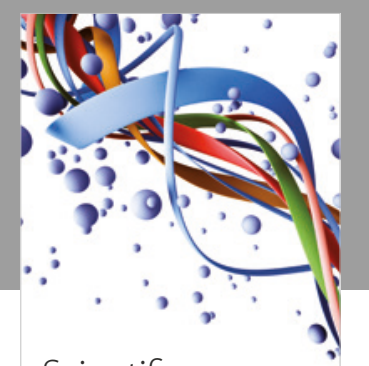

Scientifica
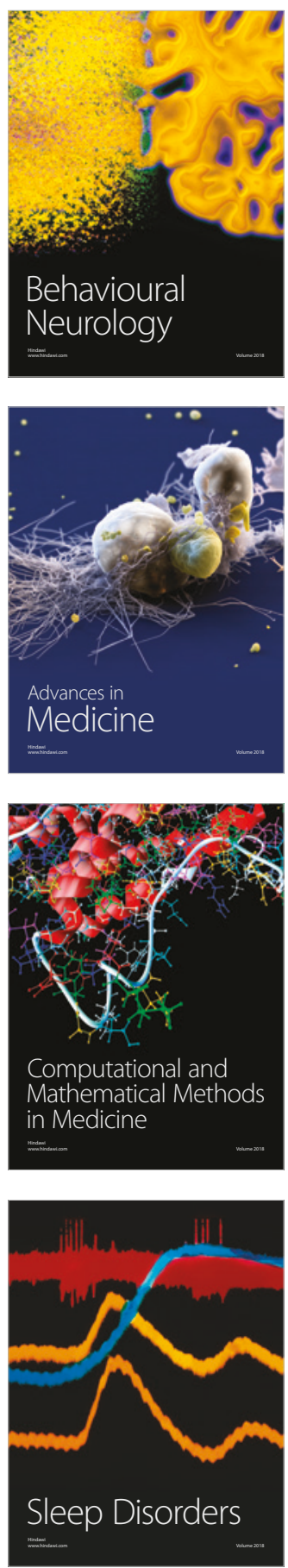

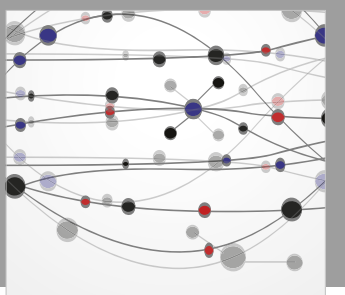

The Scientific World Journal

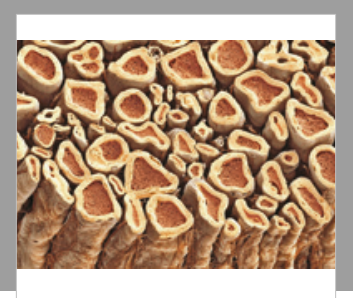

Case Reports in

Neurological Medicine

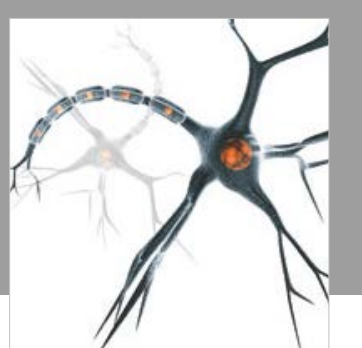

Neural Plasticity

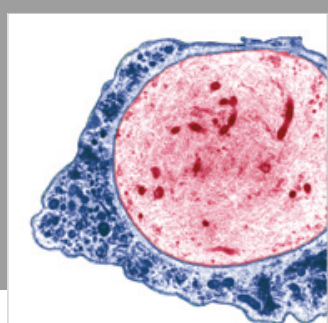

Multiple Sclerosis

International

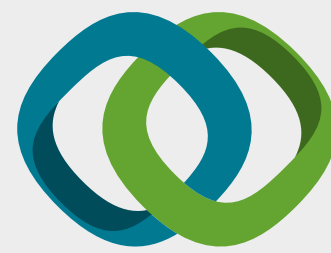

Hindawi

Submit your manuscripts at

www.hindawi.com
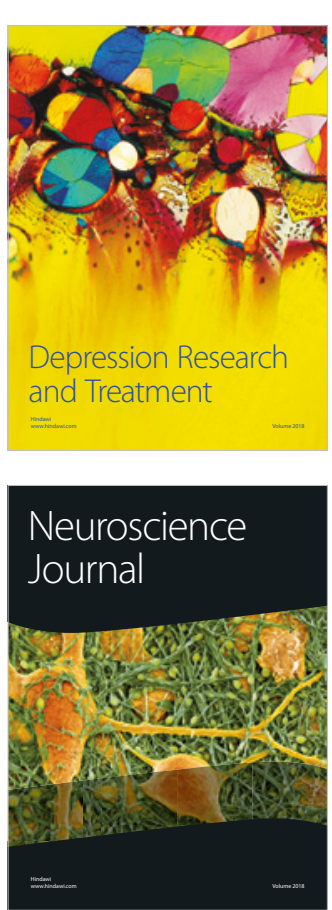

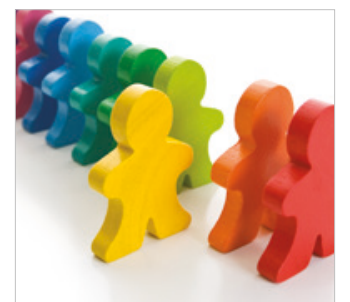

Autism

Research and Treatment
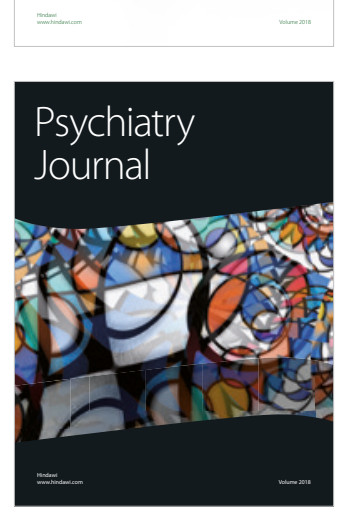
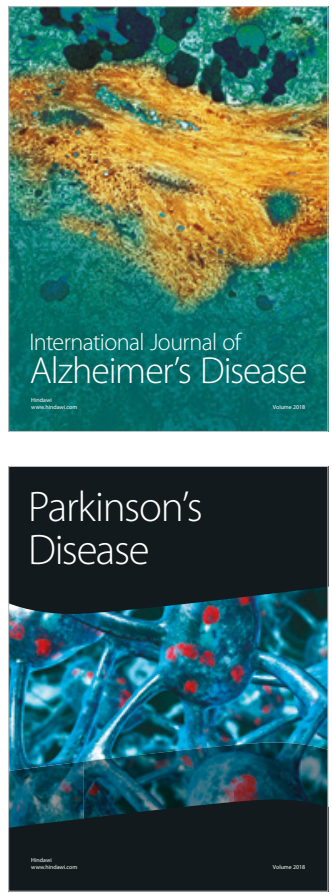
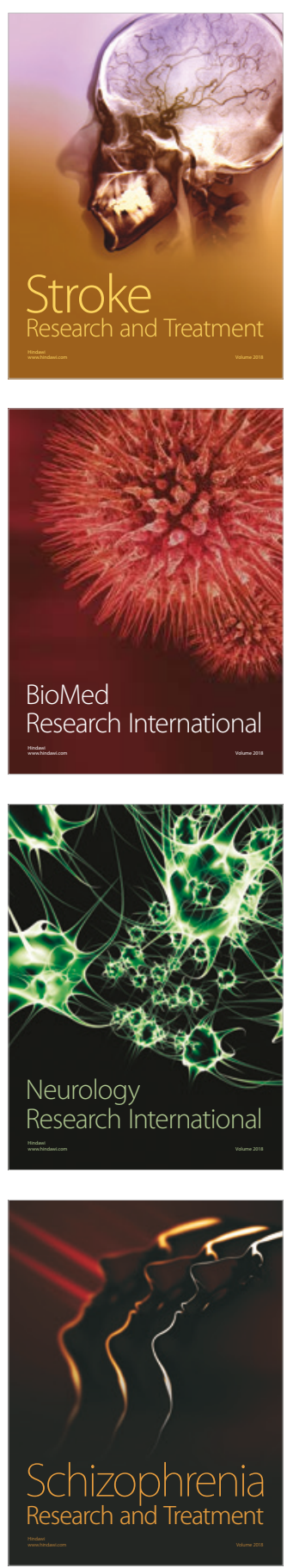